\title{
Deciphering the ins and outs of SARS-CoV- 2-specific T cells
}

\author{
Comprehensive analysis of specific and cross-reactive SARS-CoV-2 epitopes reveals functional T cell responses \\ against specific viral regions in essentially all convalescent individuals and a majority of unexposed donors, \\ demonstrating that cross-reactive responses to COVID-19 are widespread.
}

\section{André Perez-Potti, Joshua Lange and Marcus Buggert}

$\mathrm{t}$ is evident from a growing body of
research that both CD $4^{+}$and $\mathrm{CD} 8^{+}$
$\mathrm{T}$ cells specific to SARS-CoV- 2 arise following COVID-19 and can be sensitively detected during active infection and convalescence $^{1-5}$. Ex vivo stimulation of patient's immune cells with peptides derived from SARS-CoV-2 has allowed for the characterization of patient $\mathrm{T}$ cell responses that are specific to this virus. Moreover, several studies have provided evidence that a proportion of pre-existing or 'cross-reactive' $\mathrm{T}$ cells may have arisen from a previous infection with common cold coronaviruses (CCCs $)^{1-3,5}$. In the current issue of Nature Immunology, Nelde et al. ${ }^{6}$ further characterize HLA-specific peptide targets from SARS-CoV-2 in convalescent patients and identify cross-reactive epitopes probably induced by CCCs in non-infected individuals.

To elicit $\mathrm{T}$ cell responses, foreign peptides must be loaded correctly onto HLA molecules and be presented to T cells, initiating their activation and expansion. Each individual expresses a distinct set of HLA molecules, and their structural heterogeneity dictates their affinity for given peptide structures. Thus, such diversity in the HLA pool must be carefully considered in peptide design to select sequences that generate appropriate immune responses for all of the multiple HLA combinations or 'allotypes' in the population. In COVID-19 studies undertaken thus far, overlapping peptide sequences or 'pools' derived from the entire SARS-CoV-2 genome have, to a large extent, been used to study $\mathrm{T}$ cell responses in vitro, eliciting broad and HLA-indiscriminate responses. Here, Nelde and colleagues further delineate the set of SARS-CoV-2 epitopes recognized by the most common allotypes in humans, and they attempt to define immunodominant peptides at a population level. To do this, the machine learning algorithms, NetMHCpan and SYFPEITHI were
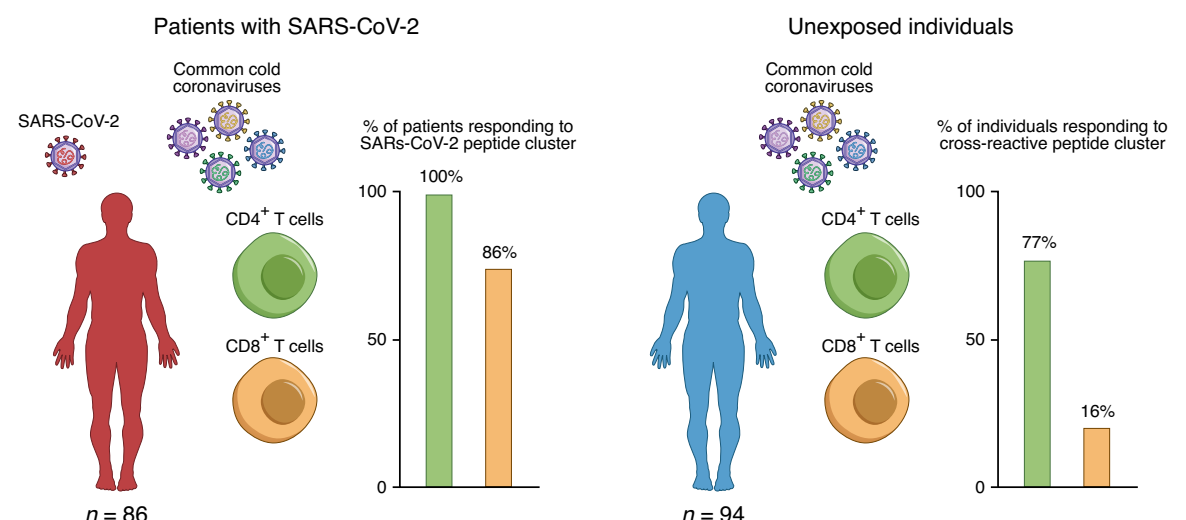

Fig. 1 | Frequency of T cell responses to SARS-CoV-2 peptides in COVID-19 convalescent and unexposed individuals. The frequency of SARS-CoV-2-specific and cross-reactive CD4+ and CD8 $8^{+} \mathrm{T}$ cell responses in convalescent individuals (left) and unexposed donors (right), respectively.

employed. These platforms draw on databases of known peptide sequences and their binding affinities for given HLA molecules, allowing for the prediction of putative allotype-specific peptides. From the analysis of all of the open reading frames (ORFs) of SARS-CoV-2, Nelde et al. selected 100 HLA class I- and 20 HLA class II (HLA-DR)-predicted peptide sequences, accounting for $10 \%$ of the virus proteome, ensuring that at least one HLA allotype was represented for $>97 \%$ of the world's population.

Functional assays revealed that $29 \%$ of the screened HLA class I and $100 \%$ of HLA-DR-predicted peptides induced T cell responses in SARS-CoV-2 convalescent and unexposed donors. In line with previous studies $^{2,4,5}$, epitope-specific CD4 ${ }^{+} \mathrm{T}$ cells were generally more pronounced and polyfunctional than epitope-specific $\mathrm{CD}^{+}$ $\mathrm{T}$ cells, on the basis of the expression of the inflammatory cytokines IFN- $\gamma$ and TNF, as well as the cytotoxicity marker CD107a. Using this approach, Nelde and colleagues identified a range of dominant peptides that generated responses in at least $50 \%$ of the patients with SARS-COV-2 that were tested. This approach aimed to characterize ORF-targeted responses in convalescent patients, shedding light on HLA-allotype-specific responses elicited by both structural and non-structural viral proteins. For instance, ORF9 (nucleoprotein) and ORF1 contained the most HLA class I-dominant peptides, and the highest recognition frequency (positive patients/total patients tested) was $83 \%$ for an ORF1 peptide. Meanwhile, most dominant HLA-DR-binding peptides corresponded to ORF9, but the highest recognition frequency was for an ORF5 peptide, at 95\%.

Activation, differentiation and expansion of virus-specific $\mathrm{CD} 4^{+} \mathrm{T}$ cells is critical to license $\mathrm{B}$ cells to produce neutralizing antibodies. The authors found anti-SARS-CoV-2-specific IgG responses against the $\mathrm{S} 1$ spike region in $84 \%$ of convalescent donors, with $16 \%$ being borderline or undetectable. Moreover, 62\% of the antibody non-responders did not have anti-SARS-CoV-2-specific IgGs against 
the nucleocapsid protein in a second test, demonstrating that $10 \%$ of the convalescent donors did not show virus-specific antibody responses by conventional serological assays. Instead, the authors demonstrated that, with their allotype-specific peptide assays, T cell responses provide enhanced sensitivity, and they were able to detect SARS-CoV2-specific responses in 10 out of the 18 donors who were antibody double negative.

In addition to convalescent individuals, the authors tested a cohort of unexposed individuals to define the levels of pre-existing and cross-reactive SARS-CoV-2 immunity that arise from previous CCC infections. A number of other labs have reported the prevalence of $\mathrm{T}$ cell reactivity in unexposed individuals to be $20-50 \%^{1-3,5}$. At first, these findings may seem surprising; however, cross-reactivity is a well-described phenomenon. Multiple viruses have been shown to provide heterologous immunity to a similar pathogen ${ }^{7,8}$. Similarly, a recent study showed that pre-existing $\mathrm{CD} 4^{+} \mathrm{T}$ cells cross-reactive to SARS-CoV-2 epitopes demonstrate comparable affinity for CCCs ${ }^{9}$. Nelde et al. showed that $31 \%$ of the HLA class I- and $70 \%$ of HLA-DR-validated SARS-CoV-2-derived peptides that showed reactivity in infected individuals were also recognized by unexposed individuals, with ORF1-derived HLA class I and ORF8-derived HLA-DR T cell epitopes showing the highest degree of cross-reactivity. Meanwhile, responses to commonly recognized epitopes from ORF5 and ORF10 in exposed individuals were not recognized by unexposed individuals. These divergent responses between exposed and unexposed individuals could partially explain the varying levels of cross-reactive responses reported in the other studies ${ }^{4,10}$, since the peptide pools used for earlier studies focused on structural proteins, such as ORF2 (spike), ORF4 (envelope), ORF5 (membrane) and ORF9 (nucleoprotein), rather than non-structural proteins, such as ORF1. Cross-reactive peptides were analyzed for sequence and physico-chemical similarities to the four CCCs (OC43, 229E, NL63 and HKU1), revealing that $70 \%$ of these cross-reactive peptides share some degree of similarity with peptides from other coronaviruses.

Next, the authors generated HLA class I and HLA-DR epitope clusters
(ECs) comprising SARS-CoV-2-specific peptides that were detected only in infected individuals or cross-reactive peptides that were tested in a second cohort of individuals infected with SARS-CoV-2 and unexposed individuals. In the unexposed group, $81 \%$ of individuals showed reactivity to the cross-reactive ECs. Such cross-reactive responses appear to be driven by HLA-DR-derived peptides, showing that $77 \%$ of the donors had $\mathrm{CD} 4^{+} \mathrm{T}$ cell responses as compared to the $16 \%$ that responded to HLA class I T cell epitopes. As a consequence, the authors conclude that $81 \%$ of the tested individuals from the unexposed group had responses to cross-reactive peptides (Fig. 1).

Evidence gathered in this study, together with recent similar findings, clearly highlights the prevalence of pre-existing SARS-CoV-2 cross-reactive T cells in the general population. One of the remaining, and perhaps the most difficult, questions to answer in SARS-CoV-2 immunity research is the role of such cross-reactive T cell responses. Do pre-existing $\mathrm{T}$ cells become activated during SARS-CoV-2 infection, and do they contribute to protection or pathology? Although unable to define such a role in this study, the authors provide important evidence that these $\mathrm{T}$ cells are functional, producing cytokines in response to SARS-CoV-2-derived peptide sequences. Importantly, there was an apparent association between the number of peptides recognized by patients and the degree of disease severity, whereby more frequent recognition of peptides was correlated with more mild symptoms. In other words, this is one of the first studies to indicate that a more polyclonal $\mathrm{T}$ cell response may be critical in protection against SARS-CoV-2. This study also brings into question the contribution of an individual's HLA allotypes to shaping the diversity of the $\mathrm{T}$ cell repertoire during both CCC and SARS-CoV-2 infections. Are HLA allotypes correlated with disease severity or outcome? How do cross-reactive T cells generated by certain allotypes during CCC infection shape the outcomes of subsequent infection with SARS-CoV-2? Or can cross-reactive $\mathrm{T}$ cells be harmful, perhaps contributing to exacerbated immune activation and hyperinflammation in severe COVID-19?
Another important consideration is that most of these studies have been performed in blood. A wealth of data indicates that circulating $\mathrm{T}$ cells do not fully recapitulate the $\mathrm{T}$ cell repertoire that targets viral infections in tissues ${ }^{11}$. Instead, resident memory $\mathrm{T}$ cells are critical immune mediators at peripheral sites, in both lymphoid and non-lymphoid tissues. As such, it would be important to investigate the levels of cross-reactivity at those sites and to define their role in SARS-CoV-2 immunity. Given that such responses appear to be driven by $\mathrm{CD} 4^{+} \mathrm{T}$ cells, which have previously been shown to induce cross-reactive protective immunity in SARS and MERS infection models in the airways ${ }^{12}$, it will be important to identify whether cross-reactive SARS-CoV2 -specific $\mathrm{CD}^{+} \mathrm{T}$ cells exist in the respiratory tract. Finally, given that $\mathrm{CD}^{+}$ $\mathrm{T}$ cells are involved in mounting humoral responses, it would be interesting to examine their functionality at the infection site and in the corresponding draining lymph nodes and to evaluate their capacity to prime B cell follicles. This would allow us to fully understand their role in launching rapid humoral immune responses against SARS-CoV-2.

\section{André Perez-Potti, Joshua Lange $\mathbb{D}$ and} Marcus Buggert (D) $₫$

Center for Infectious Medicine, Department of Medicine Huddinge, Karolinska Institutet, Karolinska University Hospital Huddinge, Stockholm, Sweden.

$凶_{e-m a i l: m a r c u s . b u g g e r t @ k i . s e}$

Published online: 26 November 2020 https://doi.org/10.1038/s41590-020-00838-5

References

1. Braun, J. et al. Nature 587, 270-274 (2020).

2. Grifoni, A. et al. Cell 181, 1489-1501.e15 (2020).

3. Le Bert, N. et al. Nature 584, 457-462 (2020).

4. Peng, Y. et al. Nat. Immunol. 21, 1336-1345 (2020).

5. Sekine, T. et al. Cell 183, 158-168.e14 (2020).

6. Nelde, A. et al. Nat. Immunol. https://doi.org/10.1038/s41590 020-00808-x (2020).

7. Turtle, L. et al. J. Exp. Med. 213, 1331-1352 (2016).

8. Wilkinson, T. M. et al. Nat. Med. 18, 274-280 (2012).

9. Mateus, J. et al. Science 370, 89-94 (2020).

10. Ferretti, A. P. et al. Immunity https://doi.org/10.1016/j. immuni.2020.10.006 (2020).

11. Buggert, M. et al. Sci. Immunol. 3, eaar4526 (2018).

12. Zhao, J. et al. Immunity 44, 1379-1391 (2016).

Competing interests

The authors declare no competing interests. 